\title{
Changes in the medium-range order during crystallization of aluminosilicate zeolites characterized by high-energy X-ray diffraction technique
}

\author{
Toru WAKIHARA, ${ }^{\#}$ Yasuhiro SUZUKI, Wei FAN, Seijiro SAITO, Shinji KOHARA, \\ Gopinathan SANKAR, ${ }^{* *}$ Manuel SANCHEZ-SANCHEZ, ${ }^{* *, \S}$ Masaru OGURA \\ and Tatsuya OKUBO ${ }^{\dagger}$
}

Department of Chemical System Engineering, The University of Tokyo, 7-3-1, Hongo, Bunkyo-ku, Tokyo 113-8656

*Japan Synchrotron Radiation Research Institute and CREST-JST, Kouto, Sayo, Hyogo 679-5198

${ }^{* *}$ Davy Faraday Research Laboratory, The Royal Institution of GB, 21 Albemarle Street, London W1S 4BS, UK

\begin{abstract}
ZSM-5 and faujasite, which are aluminosilicate zeolites with different compositions, were synthesized without using organic structure-directing agents, and the changes in the medium-range order during the crystallization of ZSM-5 and faujasite were characterized by high-energy X-ray diffraction (HEXRD) technique. It is found that that the amorphous aluminosilicates have larger fraction of 4 membered ring (4R), compared to 6 membered ring (6R) that is the most dominant in amorphous silica. Furthermore, HEXRD measurements reveal that changes in the medium-range order are characteristic for each zeolite although crystallizations of both zeolites proceeded via a similar way: that is, the dissolution of amorphous precursors to solution followed by the nucleation and crystal growth of zeolite from the soluble species. In ZSM-5 crystallization, no major changes in the medium-range order are observed although slight decrease of the fraction of $4 R$ is confirmed. On the other hand, clear changes in the medium-range order, especially on the ordering of $4 R$ and $6 R$ are confirmed in faujasite crystallization.
\end{abstract}

(02009 The Ceramic Society of Japan. All rights reserved.

Key-words : Zeolite, X-ray, diffraction, Crystallization, Crystal growth

[Received November 5, 2008; Accepted December 18, 2008]

\section{Introduction}

Zeolites are hydrated, crystalline tectoaluminosilicate that are constructed from $\mathrm{TO}_{4}$ tetrahedra $(\mathrm{T}=$ tetrahedral atom, e.g. $\mathrm{Si}$ and $\mathrm{Al}){ }^{1)-4)}$ They contain nanometer-sized and well-ordered void spaces in their structures. Because of their well-defined structures and large ion-exchange capabilities, they have been used as ion exchangers, adsorbents, catalysts, molecular-sieving membranes and so on. Most of zeolites are synthesized under hydrothermal conditions. After $\mathrm{Si}$ and $\mathrm{Al}$ sources are mixed, hydrogels obtained are heated at prescribed temperatures and crystallized into zeolites. In the case of the synthesis of zeolites with high $\mathrm{Si} /$ Al ratio, organic structure-directing agents (SDAs) are often added. The crystallization mechanisms of zeolites, however, have not been fully understood yet since crystallization of zeolites are known to be affected by various factors (e.g. starting composition, $\mathrm{pH}$, temperature, mixing procedure, agitation, synthesis time and so on). Therefore, the elucidation of the crystallization

\footnotetext{
Corresponding author: T. Okubo; E-mail: okubo@chemsys.t.utokyo.ac.jp

\# Present Address: Department of Environmental and Information Sciences, Yokohama National University, 79-7, Tokiwadai, Hodogaya-ku, Yokohama 240-8501, Japan

$\S$ Present Address: Departamento de Ingeniería Químicay Ambiental, Universidad Rey Juan Carlos, C/Tulipán, s/n 28933 Móstoles, Madrid, Spain

* Present Address: Institute of Industrial Science, The University of Tokyo, 4-6-1, Komaba, Meguro-ku, Tokyo 153-8505, Japan
}

mechanisms is still one of the major topics in zeolite science. In this study, we focus on the crystallization of ZSM-5 and faujasite. ZSM-5 and faujasite are aluminosilicate zeolites that contain three-dimensional void spaces within their structures, and have been widely utilized in industrial fields such as adsorbents and catalysts. The framework structures of ZSM-5, faujasite and amorphous silica are shown in Fig. 1. The structure of ZSM-5 consists of 5 membered ring (5R, the ring structure is denoted as $n \mathrm{R}$, where $n$ is the $\mathrm{O}$ number in the ring structure) units that are linked to form pentasil chains, and mirror images of these chains are connected via oxygen bridges to form corrugated sheets with 10R. On the other hand, the structure of faujasite consists of sodalite cages that are connected with forming double 6R (D6R). Note that faujasite whose $\mathrm{Si} / \mathrm{Al}$ ratio is larger than 1.5 is called as zeolite $\mathrm{Y}$, and that smaller than 1.5 is as zeolite X. Figure 1 also shows the relative fractions of various ring structures present in ZSM-5, faujasite and amorphous silica, ${ }^{5)}$ respectively, indicating that crystalline zeolites have smaller rings than amorphous silica (largest fraction in ZSM-5 and faujasite is $5 R$ and $4 R$, respectively, whereas that in amorphous silica is 6R).

Several studies on the aluminosilicate precursors of ZSM-5 and faujasite have been reported so far. Silicalite-1, which is pure silica ZSM-5, is the only zeolite of which whole crystallization mechanism has been deeply studied. ${ }^{6)-8)}$ The study on the crystallization process of nanosized silicalite- 1 from a clear solution in the presence of tetrapropylammonium ion $\left(\mathrm{TPA}^{+}\right)$by employing in situ small angle X-ray scattering (SAXS) and wide angle X-ray scattering (WAXS) has shown that the bimodal nanosized precursors are 
formed in the initial stage of crystallization, and they are consumed during the nucleation and crystal growth processes. ${ }^{8)}$ More recently, a study ${ }^{7)}$ on the crystallization of silicalite-1 at room temperature using SAXS and high resolution transmission electron microscopy (HRTEM) has revealed that nanosized precursor particles evolve to zeolite crystals through several intermediate states that can contribute to an aggregative growth. The top surface structure of faujasite was observed by HRTEM. ${ }^{9)}$ Based on the observation, it was proposed that $6 \mathrm{R}$ is the key building unit for the crystallization of faujasite (zeolite Y). It was found that the steps of $c a .1 .43 \mathrm{~nm}$ high on the top surface of faujasite by atomic force microscopy (AFM), which also supports that $6 \mathrm{R}$ or D6R is the key building unit for the crystallization of faujasite (zeolite Y). ${ }^{10), 11)}$ Raman spectroscopy was applied to investigate the crystallization of faujasite (zeolite $\mathrm{Y}$ and zeolite $\mathrm{X}$, respectively). ${ }^{12), 13)}$ It was found that that $6 \mathrm{R}$ connected by $4 \mathrm{R}$ or $4 \mathrm{R}$ connected by $6 \mathrm{R}$ to form sodalite cages is the mechanism for nucleation of zeolite $\mathrm{Y}$ or zeolite $X$, respectively. Recently, low-frequency vibrations in aluminosilicate species and zeolite crystals (including faujasite) were characterized by high-resolution inelastic neutron scattering and changes in the glassy state during zeolite crystallization were discussed. ${ }^{14)}$

Although these studies uncovered the crystallization of ZSM-5 and faujasite, they could not provide information regarding the changes in the atomic arrangement from amorphous to crystalline phase. Local structures can be determined by $\mathrm{NMR}^{15), 16)}$ and extended X-ray absorption spectroscopic (EXAFS) techniques; ${ }^{17), 18)}$ however, the structural information obtained by these methods is not sufficient to determine the nature of ring-structures (usually referred as sub-units) that is in the medium-range order. Diffraction methods (either X-ray or neutron ) are commonly used to obtain the atomic arrangement ${ }^{19), 20)}$ of disordered materials. Although neutron diffraction is a valuable technique to obtain sim-

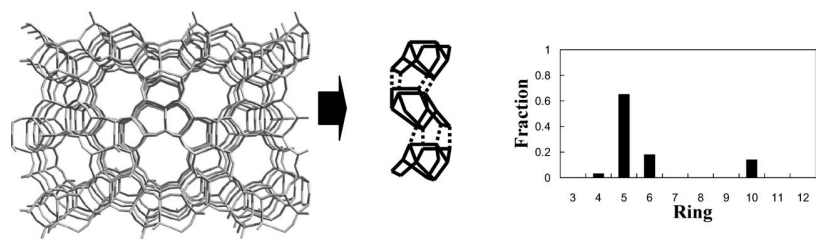

ZSM-5

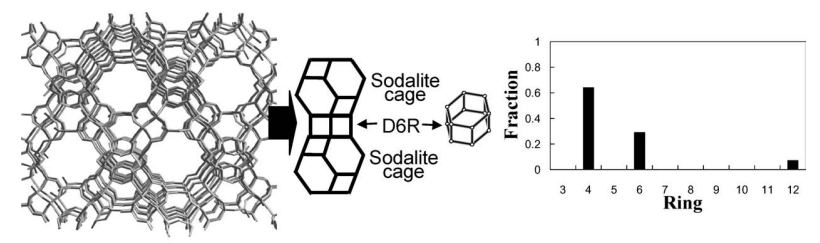

Faujasite


Amorphous silica

Fig. 1. On the left, structures of ZSM-5, faujasite and amorphous silica are shown. In the middle, building units of each structure are shown. The bar chart on the right shows the relative fractions of various ring structures present in ZSM-5, faujasite and amorphous silica. These fractions were calculated from the crystal structures for ZSM-5 and faujasite, and Reverse Monte Carlo snapshot for amorphous silica. ${ }^{5)}$ ilar information, it is mainly sensitive for $\mathrm{O}-\mathrm{O}$ correlations and in addition a small amount of hydrogen present in sample uncertainties in the data normalization. ${ }^{21)} \mathrm{X}$-ray diffraction is well suited, in particular for extracting the $\mathrm{T}-\mathrm{T}(\mathrm{T}$ : $\mathrm{Si}$, or $\mathrm{Al})$ correlations, which are very important in determining the medium-range order of zeolite precursors. In order to analyze the structure of disordered materials using diffraction methods, it is necessary to obtain the structure factor, $S(Q)$, in a wide scattering vector $Q(Q=4 \pi \sin \theta / \lambda, \theta$ : scattering angle, $\lambda$ : wavelength of photons or neutrons), since the resolution in the real space function depends on the $Q_{\max }$ of the Fourier transformation of $S(Q)$. It is essential, therefore, to conduct measurements at high energies (very low wavelength of $c a .0 .2 \AA$, which is now possible using the third generation synchrotron radiation sources). Recently, Wakihara et al. ${ }^{22)}$ and Inagaki et al. ${ }^{23), 24)}$ have reported the application of the HEXRD method for the determination of the structure of the amorphous solid produced in the initial stage of zeolite crystallization. In this study, ZSM-5 and faujasite, which are both aluminosilicate zeolites with different compositions, are synthesized without using SDAs, and the changes in the atomic arrangement from amorphous to crystalline phases are characterized by HEXRD technique.

\section{Experimental section}

\subsection{Preparation of amorphous aluminosilicates and crystalline zeolites}

ZSM-5: The aluminosilicate solution was prepared by dissolving silica particles $\left(\mathrm{SiO}_{2}, \mathrm{KE}-\mathrm{P} 10\right.$, particle size of $c a .100$ nm, Nippon Shokubai Co., Ltd.), sodium silicate $\left(\mathrm{Na}_{2} \mathrm{SiO}_{4}, \mathrm{SiO}_{2}\right.$ 51-61\%, $\mathrm{Na}_{2} \mathrm{O}$ 17-23\%, Wako Pure Chemical Industries Ltd.), aluminum hydroxide $\left(\mathrm{Al}(\mathrm{OH})_{3}\right.$, Sigma-Aldrich, Inc.) and sodium hydroxide $(\mathrm{NaOH}$, Wako Pure Chemical Industries Ltd.) in distilled water. The composition of the aluminosilicate solution was $50 \mathrm{SiO}_{2}: 1.0 \mathrm{Al}_{2} \mathrm{O}_{3}: 7.5 \mathrm{Na}_{2} \mathrm{O}: 1300 \mathrm{H}_{2} \mathrm{O}$. First, $0.18 \mathrm{~g}$ of $\mathrm{NaOH}$ and $0.087 \mathrm{~g}$ of $\mathrm{Al}(\mathrm{OH})_{3}$ were dissolved in $13.0 \mathrm{~g}$ of distilled water, and stirred for $10 \mathrm{~min}$. Then, $1.33 \mathrm{~g}$ of silica particles and $0.595 \mathrm{~g}$ of $\mathrm{Na}_{2} \mathrm{SiO}_{4}$ were added to the solution, and stirred for $30 \mathrm{~min}$. The aluminosilicate solution obtained was heated in an oven at $473 \mathrm{~K}$, and samples were recovered periodically. In the present work the amorphous samples recovered after 1,8 and $36 \mathrm{~h}$ of hydrothermal treatment were used for HEXRD measurements. The samples obtained were filtered, washed with distilled water, and dried under vacuum at room temperature. Heating periods, phases characterized by X-ray diffraction (MacScience M03X-HF) and the chemical compositions characterized by inductively coupled plasma atomic emission spectroscopy (ICP-AES; Hitachi ICPAES P-4010) of the samples are shown in Table 1 (Samples 1-3). Morphologies of the products obtained were observed by field

Table 1. Heating Periods, Phases Obtained and the Chemical Compositions of the Samples

\begin{tabular}{cclc}
\hline Samples & $\begin{array}{c}\text { Heating } \\
\text { (hours) }\end{array}$ & \multicolumn{1}{c}{ Products } & $\begin{array}{c}\text { Compositions } \\
(\mathrm{Si}: \mathrm{Al}: \mathrm{O}: \mathrm{Na})\end{array}$ \\
\hline 1 & 1 & $\mathrm{~A}$ & $19: 1.0: 40: 1.0$ \\
2 & 8 & $\mathrm{~A}+\mathrm{C}(\mathrm{ZSM}-5)$ & $20: 1.0: 42: 1.0$ \\
3 & 36 & $\mathrm{C}(\mathrm{ZSM}-5)$ & $22: 1.0: 46: 1.0$ \\
4 & 0 & A & $1.4: 1.0: 4.8: 1.0$ \\
5 & 5 & A+C (Faujasite) & $1.4: 1.0: 4.8: 1.0$ \\
6 & 24 & C (Faujasite) & $1.2: 1.0: 4.4: 1.0$ \\
\hline
\end{tabular}

A: Amorphous, C: Crystalline

Phases were characterized using conventional XRD (Mac Science, MO3XHF22, X-ray source: $\mathrm{Cu} \mathrm{K} \alpha$ ) 
emission scanning electron microscopy (FE-SEM; Hitachi S-900).

Faujasite: The aluminosilicate solutions were prepared by dissolving sodium silicate solution $\left(\mathrm{SiO}_{2} 28-30 \mathrm{wt} \%\right.$, Kishida Chemical Co., Ltd.), aluminum powder (Al, Wako Pure Chemical Industries Ltd.) and sodium hydroxide $(\mathrm{NaOH}$, Wako Pure Chemical Industries Ltd.) in distilled water. The compositions of the aluminosilicate solution was $4.0 \mathrm{SiO}_{2}: 1.0 \mathrm{Al}_{2} \mathrm{O}_{3}: 17.2 \mathrm{Na}_{2} \mathrm{O}$ : $1113 \mathrm{H}_{2} \mathrm{O}$. First, $0.162 \mathrm{~g}$ of $\mathrm{Al}$ powder and $3.80 \mathrm{~g}$ of $\mathrm{NaOH}$ were dissolved in $60.0 \mathrm{~g}$ of distilled water, and stirred for $10 \mathrm{~min}$. The solution was filtered through a $0.2 \mu \mathrm{m}$ membrane. Then, $2.40 \mathrm{~g}$ of sodium silicate solution was added to the solution. The aluminosilicate solution obtained was aged at $298 \mathrm{~K}$ for $24 \mathrm{~h}$ and then heated in an oven at $363 \mathrm{~K}$ for prescribed periods without stirring. Samples recovered after 0, 5 and $24 \mathrm{~h}$ of hydrothermal treatment were used for HEXRD measurements. Heating periods, phases and the chemical compositions of the samples are shown in Table 1 (Samples 4-6).

\subsection{High-energy $\mathrm{X}$-ray diffraction measurement}

High-energy X-ray diffraction experiments were carried out on a horizontal two-axis diffractometer, optimized for structural measurements in glass and liquid, built at the BL04B2 highenergy monochromatic bending magnet beam line of SPring-8. A bent crystal mounted on the monochromator stage fixed at a Bragg angle of 3 degree in the horizontal plane, provides incident photon energy of $61.63 \mathrm{keV}$ (Wavelength: $0.2012 \AA$ ) using bent Si (220) crystal. Pelletized amorphous aluminosilicates and

(a)

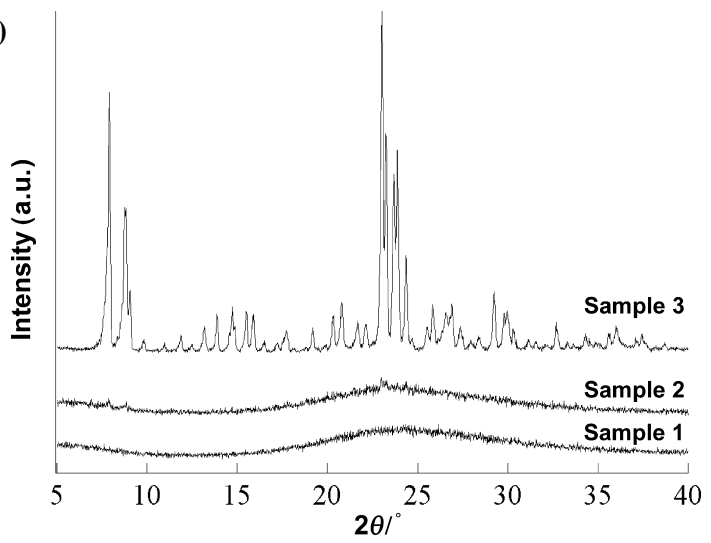

(b)

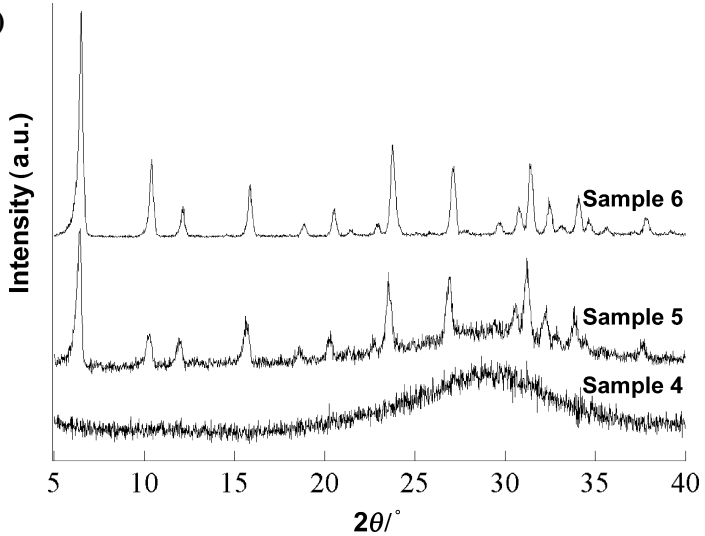

Fig. 2. XRD patterns obtained by conventional X-ray diffraction of (a) samples 1, 2 and 3 and (b) samples 4, 5 and 6, indicating that (a) ZSM-5 and (b) faujasite were formed from amorphous phase, respectively. Note that all Bragg peaks are due to ZSM-5 in (a) and faujasite in (b). crystalline zeolite samples were fixed on the sample stage and then characterized. $Q_{\max }$ collected in this study is $25 \AA^{-1}$. The collected data were subjected to well established analysis procedures including absorption, background and the Compton scattering corrections followed by normalization to the FaberZiman total structure factor, $S(Q) .{ }^{25)}$ Total correlation function, $T(r)$, is derived from eq.(1),

$$
T(r)=4 \pi \rho r+\frac{2}{\pi} \int_{Q_{\min }}^{Q_{\max }}\{Q[S(Q)-1] \sin (Q r)\} d Q
$$

where $\rho$ is the total number density. ${ }^{26)}$

\section{Results and Discussion}

Powder XRD patterns (X-ray source: $\mathrm{Cu} \mathrm{K} \alpha, 1.54 \AA$ ) of prepared samples are shown in Fig. 2. Figure 2(a) shows the XRD patterns of the products for the synthesis of ZSM-5. Slightly peaks due to Bragg diffraction are observed in sample 2, while no such peaks are seen in sample 1. Therefore, it is concluded that nucleation of crystalline ZSM-5 occurred after $c a$. $8 \mathrm{~h}$ of the hydrothermal synthesis. It is confirmed that the crystallization is completed by $36 \mathrm{~h}$ of the hydrothermal synthesis (sample 3 ). Figure 2(b) shows the XRD patterns of the products for the synthesis of faujasite. It is found that no Bragg diffraction is confirmed in sample 4. An amorphous/crystalline mixture is obtained after $5 \mathrm{~h}$ of the hydrothermal synthesis (sample 5), and the crystallization is completed by $24 \mathrm{~h}$ of hydrothermal synthesis (sample 6). To extract quantitative information from the HEXRD regarding the atomic arrangement in the amorphous and/or crystalline materials, the total correlation functions are calculated by the Fourier transformation of the total structure factor, $S(Q)$.

\subsection{Comparison of amorphous precursors of} ZSM-5 and faujasite with amorphous silica

Figure 3 shows the total correlation functions, $T(r)$ s, of amorphous ZSM-5 (sample 1), amorphous faujasite (sample 4) and amorphous silica. ${ }^{5)}$ From the $T(r) \mathrm{s}$, it is possible to identify the various distances associated with several features. The first peak in the $T(r)$ curves is related to $\mathrm{Si}-\mathrm{O}$ and $\mathrm{Al}-\mathrm{O}$ distances. Although the $Q$ range obtained here ${ }^{20)}$ is not sufficient to resolve the two distances of $c a .1 .61$ and $1.73 \AA$ for tetrahedrally coordinated $\mathrm{Si}$ and $\mathrm{Al}$ species, respectively, there is a shift in the first peak position to higher value for the systems containing larger

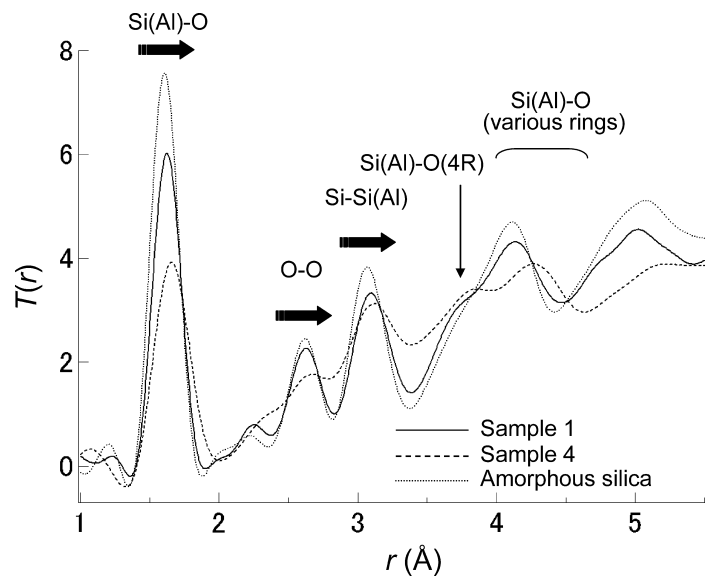

Fig. 3. Total correlation functions, $T(r)$ s, of sample 1 , sample 4 and amorphous silica. 
amounts of aluminium (sample $4>$ sample $1>$ amorphous silica). Distinct features are seen around 2.6 and $3.1 \AA$ which are due to $\mathrm{O}-\mathrm{O}$ and $\mathrm{Si}-\mathrm{Si}(\mathrm{Al})$ correlations, respectively. Similar to the peak of $\mathrm{T}-\mathrm{O}(\mathrm{Si}-\mathrm{O}$ and $\mathrm{Al}-\mathrm{O})$ at $1.61-1.73 \AA$, peak shifts to higher value can also be seen around 2.6 and $3.1 \AA$ for the systems containing larger amounts of aluminium. All these distances are closely similar to all possible ring structures and hence cannot provide any specific information required to identify the type of ring structures present in the amorphous solid as reported in the previous study. ${ }^{22)}$ The peaks in the $T(r)$ s indeed show differences in the ring structures appearing above $3.5 \AA$. Comparison of the $T(r) \mathrm{s}$ of the samples 1 and 4 with amorphous silica in the range of 3.5 and $5.5 \AA$ in Fig. 3 clearly reveals the

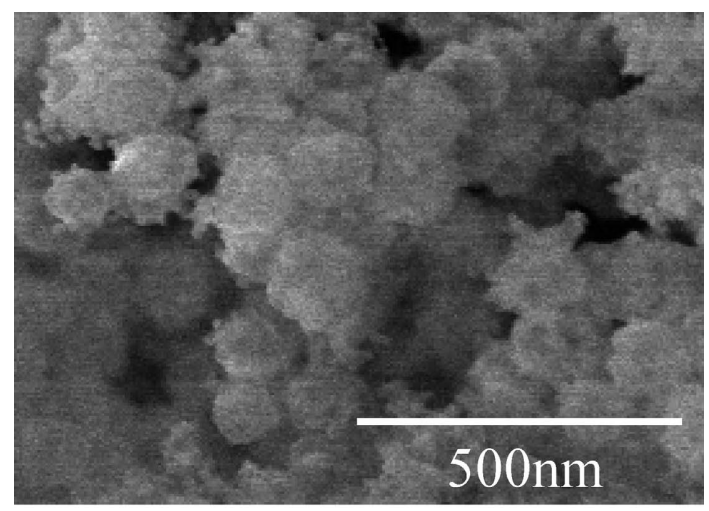

Sample 1

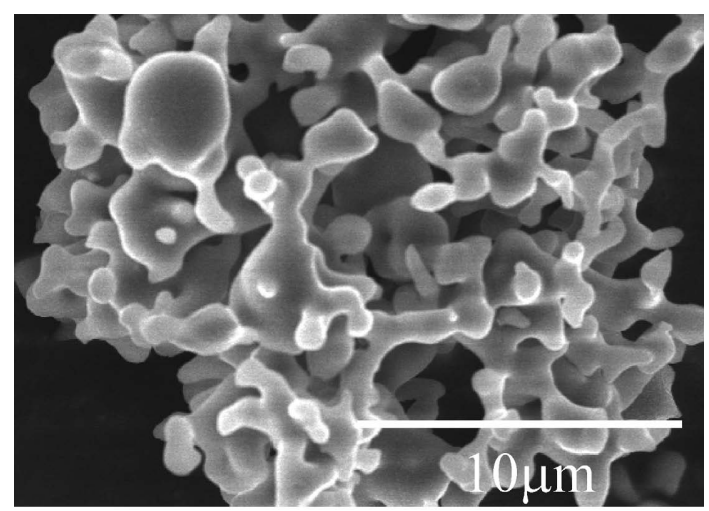

Sample 2

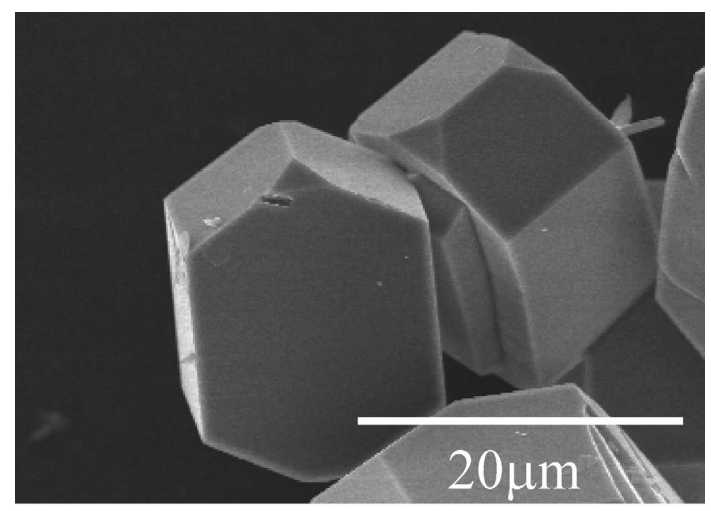

Sample 3

Fig. 4. FE-SEM image of samples 1,2 and 3. presence of different medium-range orders in these systems. It is found that the peak at $c a .3 .8 \AA$, which is mainly due to the second nearest neighbour to $\mathrm{Si}(\mathrm{Al})-\mathrm{O}$ in $4 \mathrm{R},{ }^{22)}$ is more pronounced for sample 4 (sample $4>$ sample $1>$ amorphous silica), whereas a characteristic peak at 4.2-4.3 $\AA$ which is mainly due to the ring structures larger than $4 \mathrm{R}$ is of lower amplitude and broader for sample 4 (amorphous silica > sample $1>$ sample 4). ${ }^{22)}$ The changes in these peaks indicate that Al-rich amorphous aluminosilicates have larger fraction of $4 \mathrm{R}$, compared to $6 \mathrm{R}$ that is the most dominant in amorphous silica as shown in Fig. 1. ${ }^{27)-30)}$

\subsection{Crystallization of ZSM-5}

Figure 4 shows FE-SEM images of samples 1, 2 and 3. It was found that silica particles, which are used as the Si source, are still observed after $1 \mathrm{~h}$ of hydrothermal synthesis. On the other hand, materials without specific shape (possibly amorphous matters) are observed in sample 2. In sample 3, crystalline ZSM-5 (coffin-shaped crystal) is observed. $T(r)$ s of samples $1,2,3$ and amorphous silica are shown in Fig. 5. Peaks at 1.6, $2.6 \AA$, 3.1 $\AA, 3.8 \AA$ (shoulder peak except for amorphous silica) and $4.1 \AA$ are observed. As mentioned in the previous section, the first peak $(1.6 \AA)$ in the $T(r) \mathrm{s}$ is related to $\mathrm{Si}-\mathrm{O}$ and $\mathrm{Al}-\mathrm{O}$ distances, and the peaks at $2.6 \AA$ and $3.1 \AA$ are due to $\mathrm{O}-\mathrm{O}$ and $\mathrm{Si}-\mathrm{Si}(\mathrm{Al})$ correlations. No major changes in these three peaks are observed. Peaks at $3.8 \AA$ and $4.1 \AA$ are mainly due to the second nearest neighbour to $\mathrm{Si}(\mathrm{Al})-\mathrm{O}$ in $4 \mathrm{R}$ and larger ring than $4 \mathrm{R}$, respectively. The $T(r)$ s of samples 1 and 2 are similar to each other, indicating that no major change proceeded in the medium-range order prior to the nucleation of crystalline ZSM-5. It is worth noting that $T(r)$ s of samples 1 and 2 have a shoulder peak at 3.8 $\AA$, while evident shoulder peak cannot be seen in crystalline ZSM-5 (sample 3) and amorphous silica, as shown in Fig. 5. Therefore, it is revealed that the fraction of $4 \mathrm{R}$ in the amorphous aluminosilicate is larger than that in crystalline ZSM-5 and amorphous silica, and fraction of $4 \mathrm{R}$ decreases with increasing the crystallinity. Since the morphology of amorphous matter and ZSM-5 crystals is obviously different from each other as shown in Fig. 4, and compositions of all samples are approximately the same as shown in Table 1, it appears that crystallization (at least crystal growth) proceeded via the solution-mediated transport process: that is, the soluble precursor species derived from amorphous aluminosilicate contributed to the ZSM-5 crystallization. ${ }^{3), 13), 31)}$

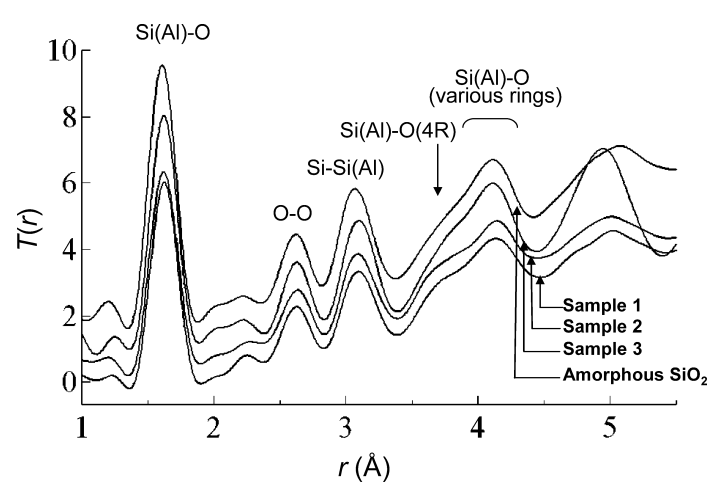

Fig. 5. Total correlation functions, $T(r) \mathrm{s}$, of amorphous silica and samples 1, 2 and 3. The functions of sample 2, sample 3 and amorphous silica are offset vertically by $0.5,1.2$ and 2 for clarity, respectively. The arrow indicates that the fraction of $4 \mathrm{R}$ in amorphous aluminosilicates are larger than that in crystalline ZSM-5 and amorphous silica (sample $1=$ sample $2>$ sample $3>$ amorphous silica). 


\subsection{Crystallization of faujasite}

Figure 6 shows FE-SEM images of samples 4, 5 and 6. Only particles in size of 100-300 nm (amorphous phase) are observed in sample 4, whereas faujasite crystals are observed among these amorphous particles in sample 5. A regular octahedron-shaped crystalline faujasite without amorphous phase is seen in sample 6, indicating that the crystallization was completed at this stage. $T(r)$ s of samples 4, 5 and 6 are shown in Fig. 7(a). Peaks are observed at 1.6, 2.6, 3.1, 3.8 and $4.2 \AA$. As mentioned in the previous section, the first three peaks are due to $\mathrm{Si}(\mathrm{Al})-\mathrm{O}, \mathrm{O}-\mathrm{O}$ and $\mathrm{Si}-\mathrm{Si}(\mathrm{Al})$ correlations, respectively. It is confirmed that peaks in the $T(r) \mathrm{s}$ are sharper with increasing the crystallinity, indicating that various distances in the crystalline phase are more rigidly distributed than that in amorphous phase. As can be seen from Fig. 7(b), a peak at $3.8 \AA$ in amorphous phase, which is mainly due to the second nearest neighbour to $\mathrm{Si}(\mathrm{Al})-\mathrm{O}$ in $4 \mathrm{R}$,
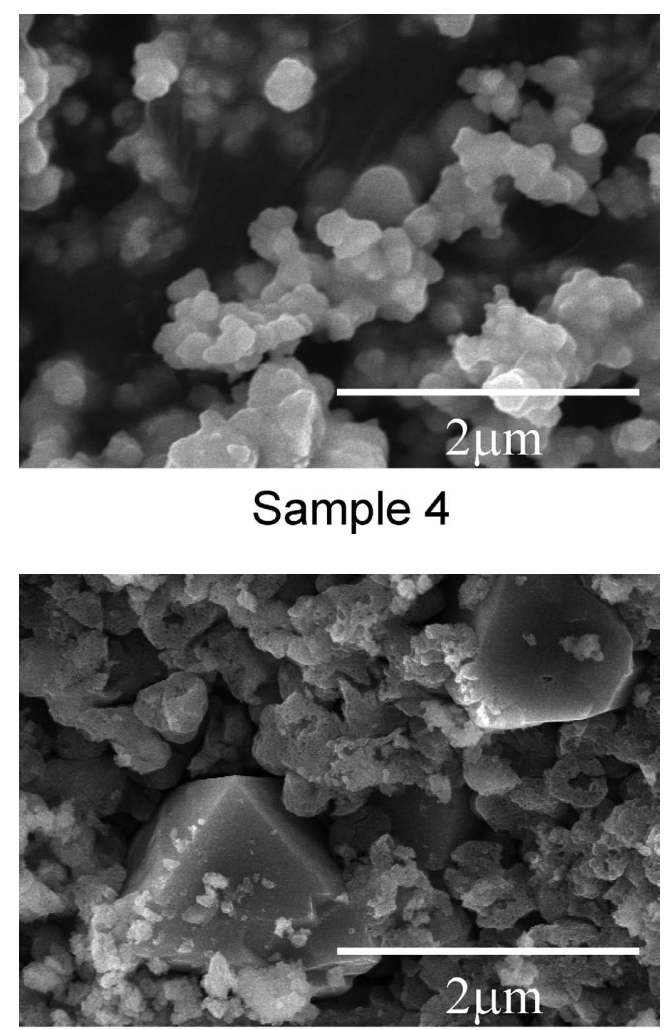

Sample 5

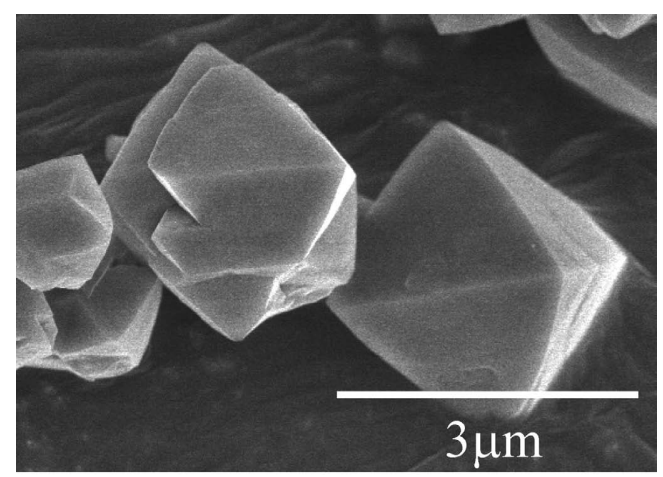

Sample 6

Fig. 6. FE-SEM images of samples 4, 5 and 6. is obviously observed; indicating that the initial amorphous gel has a large fraction of $4 \mathrm{R}$. A peak at $4.2 \AA$ in amorphous phase is mainly due to the second nearest neighbour to $\mathrm{Si}(\mathrm{Al})-\mathrm{O}$ in various rings larger than $4 \mathrm{R}$. Since the amorphous phase for the synthesis of faujasite has large fraction of $6 \mathrm{R}$ as reported in the previous study using UV-Raman spectroscopy, ${ }^{13)}$ it appears that a peak at $4.2 \AA$ is mainly due to $6 \mathrm{R}$, and the peak shift in $4.2-4.4$ $\AA$ during the crystallization is mainly due to the ordering of distorted 6R. The morphologies of amorphous aluminosilicates and crystalline zeolites are obviously different from each other as shown in Fig. 6 although there are no evident changes in the compositions before and after the crystallization as seen in Table 1. The crystallization of faujasite (at least the crystal growth), therefore, seems to proceed via the solution-mediated transport process $^{31)}$ under this condition, which is similar to the ZSM-5 crystallization shown in the previous section.

It should be noticed that the formation (ordering) of $4 \mathrm{R}$ occurs at a stage later than that of 6R, as shown in Fig. 7(b). If there is no changes in the structure of amorphous gel during the crystallization and amorphous gel is just a supplier of soluble species (if sample 5 is a mixture of sample 4 and sample 6), the difference in the peak intensities should not be observed (peak at 3.8 $\AA$ in sample 5 should be higher than that shown in Fig. 7(b)). This result shows that the structure of amorphous gel is changing towards that of the final crystalline phase during the crystallization. One possible scheme to account for these changes in medium-range order is illustrated in Fig. 7(b); first, sodalite cages, which are composed of $4 \mathrm{R}$ and $6 \mathrm{R}$, are formed in the amorphous gel although they are more distorted than those in the final crystalline phase. Then D6Rs are formed by the combination of sodalite cages (during this process six new 4Rs are formed) to form the ordered framework structure of faujasite. This scheme supports the result from a previous study using Raman spectroscopy. ${ }^{13)}$ In the case of ZSM-5 crystallization, no

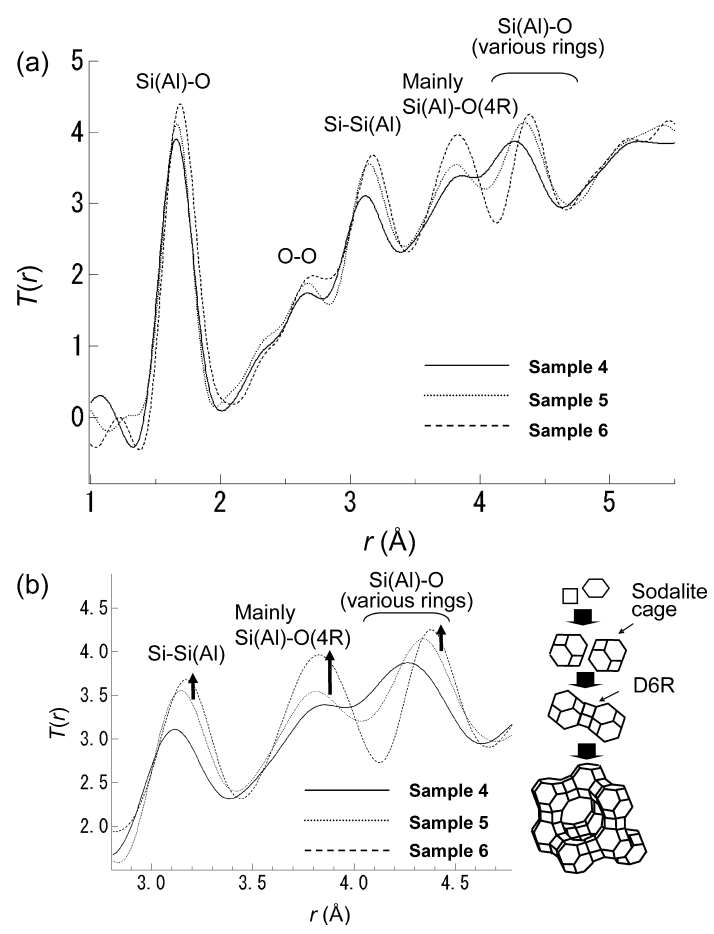

Fig. 7. (a) Total correlation functions, $T(r)$ s, of samples 4,5 and 6. (b) Magnified image of (a). One possible scheme of the formation of faujasite is shown on the right. 
major changes in the medium-range order (except for the slight decrease of the fraction of 4R) are observed. HEXRD measurements reveal that changes in the medium-range order during crystallization are characteristic for each zeolite even if both crystallizations proceeded via a similar way, such as, the dissolution of amorphous precursors to solution followed by the nucleation and crystal growth of zeolite by the soluble species.

\section{Conclusions}

Changes in the medium-range order during the crystallization of ZSM-5 and faujasite were characterized by HEXRD technique. In ZSM-5 crystallization, no major changes in the medium-range order are observed although slight decrease of the fraction of $4 \mathrm{R}$ is confirmed. On the other hand, clear changes in the medium-range order, especially on the ordering of $4 R$ and $6 R$ are confirmed in faujasite crystallization. It is difficult to obtain such information using neutron diffraction since small amounts of hydrogen introduce mainly derived from water adsorbed in porous structure uncertainties in the data normalization. ${ }^{21)}$ Furthermore, by spectroscopic method such as Raman spectroscopy, it is not possible to obtain information on the slight difference in ring structure present in amorphous/crystalline phases. X-ray diffraction, measured using a very high-energy $\mathrm{X}$-ray source, provides a better understanding of the nature of ring systems formed during the initial stages of the crystallization process of zeolites, which is applicable to any types of solids formed via amorphous intermediate. This HEXRD method in combination with Reverse Monte Carlo (RMC) modeling can provide us information on more accurate atomic arrangement of precursor particles. Such a work is now on going, and is likely to provide the structural pathway for the formation of microporous materials.

Acknowledgment This work was financially supported by Giant-in-Aid for Scientific Research (JSPS). The X-ray diffraction experiment at the SPring-8 was approved by the Japan Synchrotron Radiation Research Institute under proposal no. 2003B0157-ND1cnp.

\section{References}

1) R. M. Barrer, "Hydrothermal Chemistry of Zeolites," Academic Press, London (1982).

2) M. E. Davis and R. F. Lobo, Chem. Mater., 4, 756 (1992).

3) C. S. Cundy and P. A. Cox, Chem. Rev., 103, 663 (2003).

4) R. W. Thompson, "Molecular Sieves - Science and Technology, Synthesis, Vol. 1," Springer, Berlin, Heiderberg (1998).

5) S. Kohara and K. Suzuya, J. Phys.: Condens. Matter., 17, S77 (2005).

6) R. F. Lobo, S. I. Zones and M. E. Davis, J. Inclusion Phenom., 21, 47 (1995)
7) T. M. Davis, T. O. Drews, H. Ramanan, C. He, J. S. Dong, H. Schnablegger, M. A. Katsoulakis, E. Kokkoli, A. V. McCormick, R. L. Penn and M. Tsapatsis, Nat. Mater., 5, 400 (2006).

8) P. de Moor, T. P. M. Beelen, B. U. Komanschek, L. W. Beck, P. Wagner, M. E. Davis and R. A. van Santen, Chem.Eur. J., 5, 2083 (1999).

9) V. Alfredsson, T. Ohsuna, O. Terasaki and J. O. Bovin, Angew. Chem. Int. Ed., 32, 1210 (1993).

10) M. W. Anderson, J. R. Agger, J. T. Thornton and N. Forsyth, Angew. Chem. Int. Ed., 35, 1210 (1996).

11) T. Wakihara, A. Sugiyama and T. Okubo, Micropor. Mesopor. Mater., 70, 7 (2004)

12) P. K. Dutta, D. C. Shieh and M. Puri, J. Phys. Chem., 91, 2332 (1987).

13) G. Xiong, Y. Yu, Z. C. Feng, Q. Xin, F. S. Xiao and C. Li, Micropor. Mesopor. Mater., 42, 317 (2001).

14) G. N. Greaves, F. Meneau, O. Majerus, D. G. Jones and J. Taylor, Science, 308, 1299 (2005).

15) C. S. Cundy and P. A. Cox, Micropor. Mesopor. Mater., 82, 1 (2005).

16) M. A. Nicolle, F. Di Renzo, F. Fajula, P. Espiau and T. des Courieres, Proc. 9th Int. Zeolite Conf., Boston (1993) p.313.

17) G. Sankar, J. M. Thomas and C. R. A. Catlow, Top. Catal., 10, 255 (2000).

18) C. R. A. Catlow and G. N. Greaves, "Applications of Synchrotron Radiation," Blackie Academic and Professional, London (1990).

19) S. Kohara, K. Suzuya, K. Takeuchi, C. K. Loong, M. Grimsditch, J. K. R. Weber, J. A. Tangeman and T. S. Key, Science, 303, 1649 (2004).

20) V. Petkov, S. J. L. Billinge, S. D. Shastri and B. Himmel, $J$. Non-Cryst. Solids, 293, 726 (2001).

21) H. X. Yang, R. I. Walton, S. Antonijevic, S. Wimperis and A. C. Hannon, J. Phys. Chem. B, 108, 8208 (2004).

22) T. Wakihara, S. Kohara, G. Sankar, S. Saito, M. SanchezSanchez, A. R. Overweg, W. Fan, M. Ogura and T. Okubo, Phys. Chem. Chem. Phys., 8, 224 (2006).

23) S. Inagaki, K. Nakatsuyama, Y. Saka, E. Kikuchi, S. Kohara and M. Matsukata, J. Phys. Chem. C, 111, 10285 (2007).

24) S. Inagaki, K. Nakatsuyama, Y. Saka, E. Kikuchi, S. Kohara and M. Matsukata, Micropor. Mesopor. Mater., 101, 50 (2007).

25) T. E. Faber and J. M. Ziman, Philos. Mag., 11, 153 (1965).

26) E. A. Lorch, J. Phys. C, 2, 229 (1969).

27) P. K. Dutta and D. C. Shieh, J. Phys. Chem., 90, 2331 (1986).

28) S. Susman, K. J. Volin, R. C. Liebermann, G. D. Gwanmesia and Y. B. Wang, Phys. Chem. Glasses, 31, 144 (1990).

29) T. Uchino, A. Aboshi, S. Kohara, Y. Ohishi, M. Sakashita and K. Aoki, Phys. Rev. B: Condens. Matter, 69, 155409 (2004).

30) S. Kohara and K. Suzuya, Nucl. Instrum. Methods Phys. Res., Sect. B, 199, 23 (2003).

31) I. Kumakiri, T. Yamaguchi and S. Nakao, Ind. Eng. Chem. Res., 38, 4682 (1999). 